\section{Coffee Consumption is Associated with a Lower Incidence of Fatty Liver in Middle-aged Men}

\author{
Kazuo Funatsu, ${ }^{*}$ Takeshi Yamashita, and \\ Haruo Nakamura \\ Mitsukoshi Health and Welfare Foundation, ESTEC \\ Information-Building, 1-24-1 Nishi-shinjuku, Shinjuku- \\ ku, Tokyo 160-0023, Japan
}

(Received February 9, 2011; Accepted July 5, 2011; Published online July 8, 2011)

Whilst accumulating reports have shown the beneficial effects of coffee against chronic liver diseases, the effects of coffee against fatty liver have not yet been reported. In this cross-sectional and the followup studies, we investigated the effects of coffee on the production of fatty liver in healthy males, using ultrasonography. In the follow-up study, annual changes in daily coffee intake during the 5-year study period were compared between subjects who appeared to have fatty liver (fatty liver group) and those who did not appear to have fatty liver (non-fatty liver group) in the matched study according to age, body mass index (BMI) and daily exercise level. The effects of changes in daily coffee intake between both groups on the development of fatty liver were investigated after adjustments for related factors, such as BMI, daily coffee and alcohol intake and exercise level. In the cross-sectional study, the subjects with fatty liver were revealed to have a lower daily coffee intake than those without fatty liver. In the follow-up study, a significant difference in the changes in daily coffee intake was observed between the fatty liver and nonfatty liver groups with a decrease in daily coffee intake observed in the former group, compared with the change in the latter group. Further, daily coffee intake was negatively correlated with the development of fatty liver after adjustments for related factors. These findings suggest a protective effect of coffee against fatty liver.

\footnotetext{
*To whom correspondence should be addressed: Mitsukoshi Health and Welfare Foundation, ESTEC Information-Building, 1-24-1 Nishi-shinjuku, Shinjuku-ku, Tokyo 160-0023, Japan. Tel.: +81-3-3348-5791; Fax: +81-3-3348-5795; E-mail: funatsu@mhwf.or.jp
}

Key words — coffee, fatty liver, ultrasonography

\section{INTRODUCTION}

The prevalence of lifestyle-related diseases has been increasing in Japan ${ }^{1)}$ as a result of dietary changes, such as an increase in the composition of saturated fatty acids and alcoholic beverages and lifestyles that lead to the neglect of one's health. In response to this prevalence of lifestyle-related diseases, the number of cases with fatty liver is also escalating. ${ }^{2,3)}$ Since liver dysfunction, most cases of which are thought to be caused by fatty liver, ${ }^{4)}$ is observed very frequently in combination with obesity in annual health check-ups, fatty liver is considered to be a component of, or at least a predictor of, metabolic syndrome. ${ }^{5,6)}$

Accumulating reports have shown the beneficial effects of coffee on liver function. Epidemiologic surveys from the last 20 years have found an association between higher coffee consumption and lower levels of liver enzymes and a low risk of chronic liver diseases, including cirrhosis or hepatocellular carcinoma. ${ }^{7-12)}$ However, the beneficial effects of coffee against fatty liver have not yet been determined. In the present cross-sectional and follow-up studies, we investigated the effects of coffee on the development of fatty liver in men attending serial annual health examinations.

\section{MATERIALS AND METHODS}

Subjects — A total of 2265 male office workers employed at a single company who were between the ages of 25 and 60 years in 1999 were recruited. The workers were employed in the same service industry and had similar workloads without night duty; all the workers visited our health institution for annual health check-ups and routine physical examinations. Of these workers, 419 were excluded at the start of the study for the following reasons: 141 because of treatment for chronic liver diseases, such as chronic hepatitis, liver cirrhosis, fatty liver etc., diabetes or hyperlipidemia, and 278 because of missing information regarding data on lifestyle. During the 5-year follow-up period, 234 subjects were excluded because of the start of treatment for chronic liver diseases, diabetes and/or hy- 
perlipidemia or because of missing information regarding data on lifestyle. Therefore, 1612 subjects were finally included in the present study. Information on previous and present illnesses was obtained using a self-administered questionnaire that was completed annually.

Data Collection — Data on life style over the 5year study period were obtained by completing a self-administered questionnaire that was mailed to each subject prior to their health examination. The lifestyle items that were used in this study were the intake of alcohol, coffee and green tea and the degree of exercise. Blood chemistry tests, including liver enzyme levels, and a routine abdominal ultrasound test were performed during the morning after an overnight fast at each health check-up every year and their data obtained in 1999 and 2004 were used in the present study. The abdominal ultrasound examinations were performed by experienced radiologists using a Hitachi EUB2000 or EUB525 (Hitachi, Tokyo, Japan). The presence or absence of fatty liver was judged separately by two specialists in gastroenterology. The examiner was unaware of the blood chemistry data and the volume of daily coffee consumption.

Ultrasonographic findings showing a bright liver, an increase in the liver-kidney contrast, and/or a decrease in liver deep echo were regarded as indicative of fatty liver. This study was approved by The Ethical Committee of the Mitsukoshi Health and Welfare Foundation.

Cross-sectional Study — The relations between fatty liver finding, as observed by ultrasound test in 1999 or 2004, and such factors as daily coffee, green tea and alcohol intake, body mass index (BMI; calculated as weight in kilograms divided by the square of height in meters), exercise level and liver enzyme level (alanine aminotransferase) were investigated in a cross-sectional study.

Coffee and green tea intake was each expressed as the average daily intake of cups per day. Alcohol intake was converted into a value equivalent to one go, a traditional Japanese unit of volume for sake (one go contains about $22 \mathrm{~g}$ of ethanol), and was scored into 5 categories as follows: 1 point: no alcoholic beverages or less than 1 go per day, 2 points: between 1 and less than 2 go per day, 3 points: between 2 and less than 3 go per day, 4 points: between 3 and less than 4 go per day, 5 points: 4 or more go per day. Exercise levels were scored as follows: 1 point: almost no exercise or walking, 2 points: 20 min continuous or more than $1 \mathrm{hr}$ walking or exercise/day one day per week, 3 points: 20 min continuous or more than $1 \mathrm{hr}$ walking or exercise/day two days per week, 4 points: 20 min continuous or more than $1 \mathrm{hr}$ walking or exercise/day 3 to 4 days per week, 5 points: 20 min continuous or more than $1 \mathrm{hr}$ walking or exercise/day more than 5 days per week.

Five-Year Follow-up Study for Matched Cases_- Of a total of 1612 subjects, 1236 males were identified as not having fatty liver at the time of the initial examination in 1999. These 1236 males were divided into the two groups; those with fatty liver in $2004(n=164)$, and those without fatty liver in $2004(n=1072)$. We compared changes in daily coffee intake over the 5-year study period between those with fatty liver and without fatty liver among subjects with a similar age, BMI and exercise level. For this purpose, 2 subjects in those without fatty liver per one subject in those with fatty liver were matched according to age, BMI and exercise level in 2004; the subjects from those without fatty liver were randomly selected from amongst those subjects that met the matching criteria. A total of 492 males, consisting of the 164 males from those with fatty liver (fatty liver group) and 328 males from those without fatty liver (non-fatty liver group), were examined in this study. Serial changes in daily coffee intake from 1999 to 2004 were compared between these two matched groups. Further, the effect of changes in daily coffee intake over the 5-years study period on the development of fatty liver in these groups was investigated after adjustments for age, BMI, exercise level, daily coffee and alcohol intake in 2004, and differences in BMI, exercise level and daily alcohol intake over the 5-year study period.

Data Analysis — Unpaired $t$-tests were used to analyze the correlations between the development of fatty liver and age; BMI; exercise level; coffee, green tea and alcohol intake; smoking index, and biochemical function. Changes in daily coffee intake over the 5-year study period were compared between the fatty liver and the non-fatty liver groups using a two-way repeated measures analysis of variance (ANOVA). The effects of changes in daily coffee intake difference over the 5-year study period were also compared between the two groups using a logistic regression analysis after adjustments for age, BMI, and daily coffee and alcohol intake in 2004 as well as differences in BMI, exercise level, and daily alcohol intake over the 5-year study period. All $p$-values were two sided, and the level of 
significance was $p<0.05$.

\section{RESULTS}

\section{Cross-sectional Study}

Among the 1612 subjects, 376 (23.3\%) exhibited fatty liver in 1999 and 468 (29.0\%) exhibited fatty liver in 2004. As of 2004, fatty liver had disappeared in 72 of the 376 cases of fatty liver observed in 1999, but 164 new cases developed amongst the 1236 males without fatty liver in 1999. In both 1999 and 2004, the subjects with fatty liver reported a lower volume of daily coffee intake and exhibited a higher BMI and liver enzyme levels, compared with those without fatty liver (Fig. 1). No significant differences in the mean daily intakes of alcohol and green tea were observed between the two groups, either in 1999 or 2004.

\section{Follow-up Study for Matched Cases}

The characteristics of the subjects in 1999 and 2004 in the follow-up study for matched cases are shown in Table 1. The mean ( \pm S.D.) ages of the subjects for the non-fatty liver group and fatty liver groups in 2004 were $49.2 \pm 7.0$ and $49.4 \pm 7.6$, re-
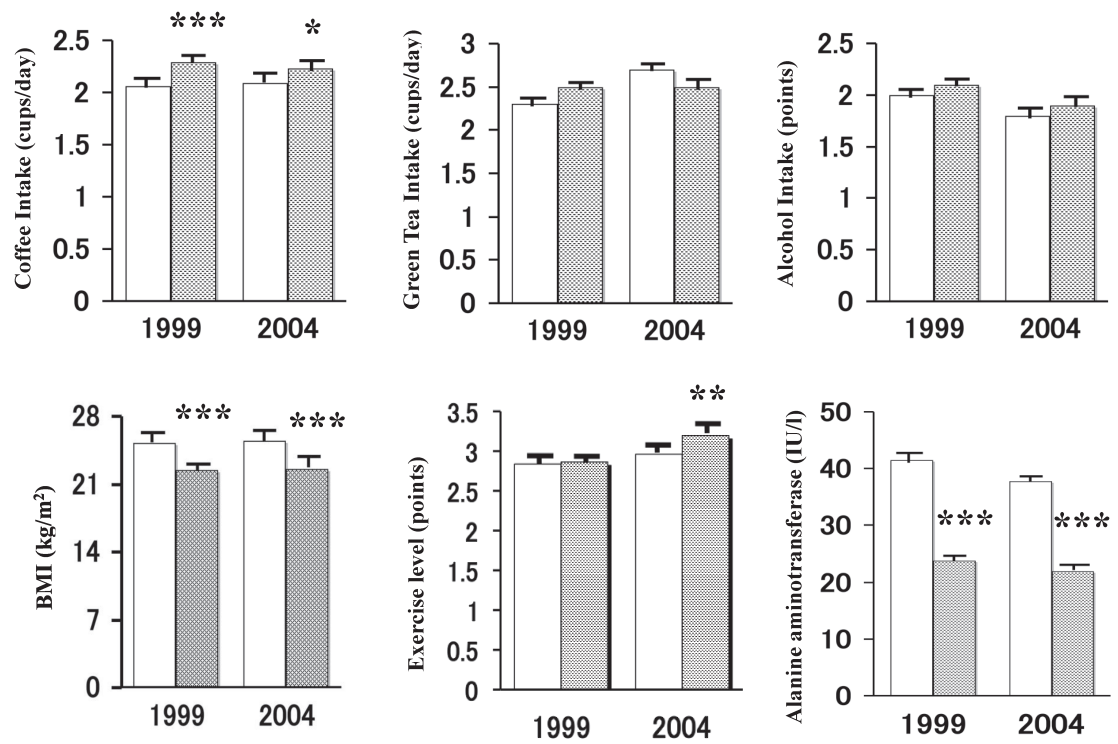

Subjects with Fatty Liver

Subjects without Fatty Liver

Fig. 1. Bar Graph Showing Daily Coffee, Green Tea and Alcohol Intake; BMI (the Weight in Kilograms Divided by the Square of the Height in Meters); Exercise Level; and Alanine Aminotransferase Level between Subjects with Fatty Liver and Subjects without Fatty Liver in 1999 and 2004

Among the 1612 subjects, 376 had fatty liver in 1999 and 468 had fatty liver in 2004. Alcohol intake and exercise level were scored into 5 categories, as mentioned in the text. Data are expressed as mean \pm S.E. ${ }^{*} p=0.06,{ }^{* *} p<0.01,{ }^{* * *} p<0.001$.

Table 1. Characteristics of the Non-fatty Liver Group and the Fatty Liver Group in 1999 and 2004 in the Matched Study

\begin{tabular}{|c|c|c|c|c|c|c|}
\hline \multirow{2}{*}{$\begin{array}{l}\text { Characteristic } \\
\text { Year }\end{array}$} & $\begin{array}{l}\text { Non-fatty } \\
\text { liver group }\end{array}$ & $\begin{array}{c}\text { Fatty liver } \\
\text { group }\end{array}$ & $p$ value & $\begin{array}{l}\text { Non-fatty } \\
\text { liver group }\end{array}$ & $\begin{array}{c}\text { Fatty liver } \\
\text { group }\end{array}$ & $p$ value \\
\hline & \multicolumn{3}{|c|}{1999} & \multicolumn{3}{|c|}{2004} \\
\hline Age (yr) & $44.2 \pm 7.0$ & $44.4 \pm \quad 7.6$ & 0.86 & $49.2 \pm$ & $49.4 \pm$ & 0.86 \\
\hline BMI $\left(\mathrm{kg} / \mathrm{m}^{2}\right)$ & $24.1 \pm$ & $24.1 \pm$ & 0.85 & $24.7 \pm$ & $25.0 \pm$ & 0.14 \\
\hline Exercise level (points) ${ }^{*}$ & $2.8 \pm$ & $2.8 \pm$ & 0.65 & $3.1 \pm$ & $2.9 \pm$ & 0.23 \\
\hline Coffee intake (cups/day) & $2.6 \pm$ & $2.6 \pm \quad 1.6$ & 0.97 & $3.0 \pm$ & $2.3 \pm$ & $<0.01$ \\
\hline Alcohol intake (points)* & $2.1 \pm$ & $2.0 \pm 13$ & 0.61 & $1.9 \pm$ & $1.8 \pm$ & 0.65 \\
\hline Green tea intake (cups/day) & $2.3 \pm$ & $2.4 \pm \quad 1.6$ & 0.53 & $2.4 \pm$ & $2.5 \pm \quad 1.7$ & 0.74 \\
\hline Smoking index & $341.8 \pm 305.8$ & $351.1 \pm 351.9$ & 0.78 & $409.8 \pm 383.6$ & $376.0 \pm 366.9$ & 0.35 \\
\hline LDL-cholesterol (mg/dl) & $111.5 \pm 28.8$ & $117.7 \pm 30.4$ & 0.03 & $122.2 \pm 28.6$ & $129.0 \pm 29.4$ & 0.02 \\
\hline Fasted blood sugar (mg/dl) & $98.6 \pm 9.3$ & $99.7 \pm$ & 0.21 & $98.9 \pm 15.3$ & $102.7 \pm 12.9$ & 0.006 \\
\hline
\end{tabular}

Plus-minus values are means \pm S.D. *Alcohol intake and exercise level are scored from 1 to 5 points, as mentioned in the text. 
Table 2. Logistic Regression Analysis to Determine the Effects of Changes in Daily Coffee Intake Difference over the 5-year Study Period on the Development of Fatty Liver in the Fatty Liver and the Non-fatty Liver Groups, with Adjustments for Related Factors, in Matched Cases

\begin{tabular}{lccc}
\hline \hline & Odds Ratio & 95\% CI & $p$ Value \\
\hline Age (2004) & 1.01 & $0.99-1.04$ & 0.33 \\
BMI (2004) & 1.01 & $0.92-1.11$ & 0.83 \\
Exercise Level (2004) & 0.957 & $0.82-1.12$ & 0.58 \\
Daily Coffee Intake (2004) & 0.736 & $0.61-0.89$ & 0.001 \\
Daily Alcohol Intake (2004) & 0.90 & $0.75-1.08$ & 0.26 \\
BMI Difference (2004-1999) & 1.34 & $1.10-1.63$ & 0.004 \\
Exercise Level Difference (2004-1999) & 0.96 & $0.83-1.11$ & 0.58 \\
Daily Alcohol Intake Difference (2004-1999) & 1.08 & $0.90-1.29$ & 0.44 \\
Daily Coffee Intake Difference (2004-1999) & 0.77 & $0.65-0.92$ & 0.004 \\
\hline
\end{tabular}

A total of 492 subjects without fatty liver in 1999 were divided into the two groups; subjects without fatty liver in 2004 (non-fatty liver group; $n=328$ ) and subjects with fatty liver in 2004 (fatty liver group; $n=164$ ), matched for age, BMI and exercise level in 2004.

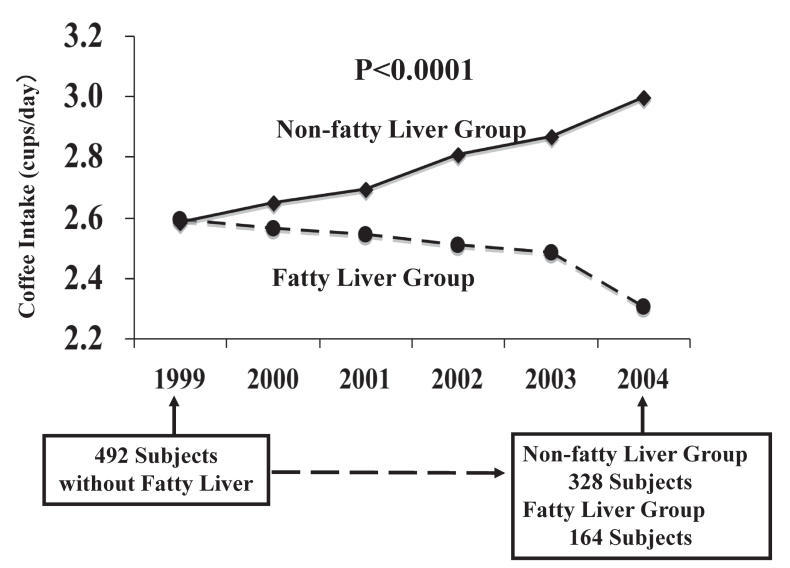

Fig. 2. Comparison of Changes in Daily Coffee Intake over the Five-Year Study Period between the Non-fatty Liver and the Fatty Liver Groups

A total of 492 subjects without fatty liver in 1999, consisting of 164 subjects with fatty liver in 2004 (fatty liver group) and 328 controls, matched according to age, BMI and exercise level in 2004 (non-fatty liver group), were followed for 5 years. The comparison was made using a two-way repeated measures ANOVA.

spectively $(p=0.86)$. The mean BMI and exercise level in 2004 were $24.7 \pm 2.0$ and $3.1 \pm 1.5$ in the non-fatty liver group, respectively, and $25.0 \pm 2.4$ ( $p=0.14, v s$. non-fatty liver group) and $2.9 \pm 1.6$ ( $p=0.23, v s$. non-fatty liver group) in the fatty liver group. There were no significant differences in age, BMI and exercise level in 2004 between the fatty liver and non-fatty liver groups. No significant differences were observed about BMI, exercise level, daily coffee, alcohol and green tea intake, and smoking index in 1999 between the non-fatty liver and the fatty liver groups. Low-density lipoprotein (LDL)-cholesterol in the fatty liver group was higher than that in the non-fatty liver group both in 1999 and 2004. Fasted blood sugar was higher in the fatty liver group, compared to the non-fatty liver group in 2004. Whilst coffee intake in the fatty liver group decreased constantly over the 5year period, it increased significantly in the nonfatty liver group (Fig. 2, $p<0.001$ ). Whilst the BMI in the fatty liver group increased over the 5-year pe$\operatorname{riod}\left(0.916 \mathrm{~kg} / \mathrm{m}^{2}\right)$, compared with that in the nonfatty liver group $\left(0.577 \mathrm{~kg} / \mathrm{m}^{2}, p=0.001\right)$, the exercise levels showed an opposite trend (non-fatty liver group, 0.221 points: fatty liver group, 0.098 points, $p=0.437$ ). No significant changes in daily alcoholic intake were observed (non-fatty liver group, -0.186 points: fatty liver group, -0.177 points, $p=$ 0.934). After adjustments for these related factors, the increase in daily coffee intake over the 5-year period in the non-fatty liver group, compared with the change in the fatty liver group, was found to be related to the protective effect of coffee against fatty liver, with an odds ratio of 0.77 [95\% confidence interval (CI), 0.65-0.92; $p=0.004$, Table 2].

\section{DISCUSSION}

In Japan, the prevalence of fatty liver is reported to be increasing as 35\% in Japanese men and 20\% in Japanese women, or about twice the values reported 10 years ago. ${ }^{2,3)}$ Many patients with nonalcoholic fatty liver disease (NAFLD) have a long and benign course; others, however, exhibit inflammation and fibrosis and progress to end-stage liver injury, such as cirrhosis, which is called as nonalcoholic steatohepatitis (NASH). NAFLD, including $\mathrm{NASH}$, are now considered to be the hepatic manifestation of the metabolic syndrome. ${ }^{13,14)} \mathrm{NASH}$ is estimated to affect $1-3 \%$ of Japanese adults and is 
considered to become the most important cause of end-stage liver disease.

Centuries of coffee drinking have made it the most consumed beverage in the world. Data on the potential beneficial effects of coffee on liver function and against liver diseases have accrued over the last two decades. Several epidemiologic studies have reported inverse associations of coffee drinking with levels of liver enzymes, ${ }^{7,15,16)}$ as well as with the risk of chronic liver diseases, ${ }^{17)}$ including liver cirrhosis ${ }^{10,18)}$ and even hepatocellular carcinoma. ${ }^{11,19,20)}$ The present epidemiological study revealed, for the first time, that coffee intake may protect against fatty liver. Since the subjects in this study were healthy individuals employed at the same company and maintained a similar lifestyle, including their work and home life, during the 5year study period, the present results were considered to be relatively reliable. The subjects in this study were limited to only men for the following reasons: first, the average prevalence of fatty liver classified according to sex was reported to be twice as high in men as it was in women in Japan;2) second, estrogen levels might affect the development of fatty liver in women, since the prevalence of fatty liver increases rapidly in post-menopausal women. ${ }^{5)}$ In this study, those subjects, who were in therapy with chronic liver diseases, diabetes mellitus and/or hyperlipidemia, were excluded, because the medication to these diseases might influence to the occurrence of fatty liver disease. The diagnosis of fatty liver is usually based on abdominal ultrasonography, which has been established as a simple, reliable and commonly used modality for the clinical screening of fatty liver. ${ }^{21)}$ Since the ultrasound equipment used to perform the examinations, the radiologists who performed the examinations, and the gastroenterology specialists who made the diagnoses were the same in 1999 and 2004, the differences between the examinations years were thought to be negligible. Since daily coffee intake varied from year to year on an individual basis, we analyzed the data using a two-way repeated measures ANOVA. A significant difference in the changes in daily coffee intake was observed between the fatty liver group and the non-fatty liver group, with a decrease in daily coffee intake observed in the former group, compared with the change in the latter group, suggesting a possible relation between decreased coffee intake and the development of fatty liver. Since the body weight, exercise level, alcohol intake, and the change in body weight, exercise level and alcohol intake over the 5-year study period between the two groups might influence the development of fatty liver, a logistic regression analysis was performed after adjusting for these factors. As a result, the increase in daily coffee intake in the non-fatty liver group, compared with the decrease in daily coffee intake in the fatty liver group, was found to possibly be related to the protection of fatty liver, with a significant odds ratio. Further, whilst increase in the BMI was related to the development of fatty liver, change in alcohol intake was not a significantly independent factor in the development of fatty liver, which is consistent with a previous report based on data obtained from health checkups. ${ }^{22,23)}$ Although alcohol is known as an important risk factor of fatty liver, the subjects in this study were unlikely to be heavy drinkers or habitual drinkers, and the effects of alcohol on liver function were thought to be relatively minimal, since they were concerned enough about their health to undergo an annual health check-up.

The presence of steatosis is an important marker of multiorgan insulin resistance. ${ }^{24)}$ In liver, insulin resistance may be related to alterations in the pathways of uptake, synthesis, degradation, or secretion in hepatic lipid metabolism. ${ }^{4)}$ Hyperinsulinemia resulting from insulin resistance increases the synthesis of fatty acids in hepatocytes by increasing glycolysis and favours the accumulation of triglycerides within hepatocytes by decreasing the hepatic production of apolipoprotein B-100, with mitochondrial oxidation overload and the production of reactive oxygen species capable of inducing lipid peroxidation of hepatocyte membranes. ${ }^{25)}$ Coffee contains a vast number of different chemicals, any one of which may be responsible for its protective effects on the liver. ${ }^{26)}$ Chlorogenic acid and its derivatives contained in coffee are known to be associated with an increase in insulin sensitivities, ${ }^{27)}$ and to have a strong protective antioxidant effect, which might protect the liver against the accumulation of lipids within hepatocytes. It was reported that caffeine might also improve insulin sensitivity and fatty liver in mice. ${ }^{28)}$ In addition, coffee and caffeine can cause weight loss, ${ }^{29,30)}$ possibly reducing visceral fat and thereby reducing lipid accumulation in hepatocytes. Of all the types of fat tissue, visceral fat is the most metabolically active and which plays an important role in the development of fatty liver. ${ }^{31)}$ The severity of fatty liver was reported to be positively correlated with visceral fat accumulation, regardless of $\mathrm{BMI}^{32}$ and excess visceral fat, 
when inflamed, has a large effect on insulin sensitivity. ${ }^{33)}$ Recently, a high consumption of caffeinecontaining coffee was reportedly associated with a higher adiponectin and lower inflammatory marker concentrations, ${ }^{34)}$ which may play a role in the beneficial effects of coffee on insulin sensitivities and the alleviation of hepatic steatosis. ${ }^{35,36)}$ However, since other caffeine-containing beverages, such as green tea or cola, failed to demonstrate a significant association between these beverages and a reduction in liver-related endpoints, the mechanisms involved and the substances in coffee that may be responsible for its effect on the liver remain to be elucidated.

A limitation of this study is the lack of information on decaffeinated coffee consumption, brewing method, and variations in cup size and strength. However, because Japanese people usually drink either instant coffee, filtered coffee or canned coffee, the constituents of which are similar to each other, the results in this study were considered to be reliable. Sugar and cream in coffee might have the potential effect on fatty liver, but they were not considered to be a risk factor for fatty liver, since coffee intake in the non-fatty liver group was higher than that in the fatty liver group. Since elevated fat accumulation in the liver was recently reported to be accompanied by atherosclerosis and metabolic syndrome ${ }^{37-39)}$ in a manner that was independent of visceral adiposity, ${ }^{40,41)}$ the protective effects of coffee against fatty liver may also protect against the development of lifestyle-related diseases. Thus, the possible protective effects of coffee against fatty liver deserve further investigation.

\section{REFERENCES}

1) Health and Welfare Statistics Association (2007) Annual Statistical Report of National Health Conditions. Journal of Health and Welfare Statistics, 54, 88-92 (in Japanese).

2) Imamura, Y., Uto, H., Oketani, M., Hiramine, Y., Hosoyamada, K., Sho, Y., Hiwaki, T., Baba, Y., Tahara, K., Kubozono, O., Kusano, K. and Tsubouchi, H. (2008) Association between changes in body composition and the increasing prevalence of fatty liver in Japanese men. Hepatol. Res., 38, 1083-1086.

3) Komeda, T. (2005) Obesity and NASH in Japan. Hepatol. Res., 33, 83-86.

4) Angulo, P. (2002) Nonalcoholic fatty liver disease. N. Engl. J. Med., 346, 1221-1231.
5) Kogiso, T., Moriyoshi, Y. and Nagahara, H. (2007) Clinical significance of fatty liver associated with metabolic syndrome. Hepatol. Res., 37, 711-721.

6) Korenblat, K. M., Fabbrini, E., Mohammed, S. and Klein, S. (2008) Liver, muscle and adipose tissue insulin action is directly related to intrahepatic triglyceride content in obese subjects. Gastroenterology, 134, 1369-1375.

7) Poikolainen, K. and Vartianen, E. (1997) Determinants of gamma-glutamyltransferase: Positive interaction with alcohol and body mass index, negative association with coffee. Am. J. Epidemiol., 146, 1019-1024.

8) Honjo, S., Kono, S., Coleman, M. P., Shinchi, K., Sakurai, Y., Todoroki, I., Umeda, T., Wakabayashi, K., Imanishi, K., Nishikawa, H., Ogawa, S., Katsurada, M., Nakagawa, K. and Yoshizawa, N. (1999) Coffee drinking and serum gammaglutamyltransferase: an extended study of SelfDefense Officials of Japan. Ann. Epidemiol., 9, 325331.

9) Freedman, N. D., Everhart, J. E., Lindsay, K. L., Ghany, M. G., Curto, T. M., Shiffman, M. L., Lee, W. M., Lok, A. S., Di Bisceglie, A. M., Bonkovsky, H. L., Hoefs, J. C., Dienstag, J. L., Morishima, C., Abnet, C. C., Sinha, R. and HALT-C Trial Group (2009) Coffee intake is associated with lower rates of liver disease progression in chronic hepatitis $\mathrm{C}$. Hepatology, 50, 1360-1369.

10) Corrao, G., Zambon, A., Bagnardi, V., D'Amicis, A. and Klatsky, A.; Collaborative SIDECIR Group (2001) Coffee, caffeine, and the risk of liver cirrhosis. Ann. Epidemiol., 11, 458-465.

11) Tanaka, K., Hara, M., Sakamoto, T., Higaki, Y., Mizuta, T., Eguchi, Y., Yasutake, T., Ozaki, I., Yamamoto, K., Onohara, S., Kawazoe, S., Shigematsu, H. and Koizumi, S. (2007) Inverse association between coffee drinking and the risk of hepatocellular carcinoma: a case-control study in Japan. Cancer Sci., 98, 214-218.

12) Ruhl, C. E. and Everhart, J. E. (2005) Coffee and caffeine consumption reduce the risk of elevated serum alanine aminotranferase activities in the United States. Gastroenterology, 128, 24-32.

13) Pagano, G., Pacini, G., Musso, G., Gambino, R., Mecca, F., Depetris, N., Cassader, M., Cavallo-Perin, P. and Rizzetto, M. (2002) Nonalcoholic steatohepatitis, insulin resistance, and metabolic syndrome: further evidence for an etiologic association. Hepatology, 35, 367-372.

14) Targher, G. (2007) Non-alcoholic fatty liver disease, the metabolic syndrome and the risk of cardiovascular disease: the plot thickens. Diabet. Med., 24, 
1-6.

15) Casiglia, E., Spolaore, P., Ginocchio, G. and Ambrosio, G. B. (1993) Unexpected effects of coffee consumption on liver enzymes. Eur. J. Epidemiol., 9, 293-297.

16) Honjo, S., Kono, S., Coleman, M. P., Shinchi, K., Sakurai, Y., Todoroki, I., Umeda, T., Wakabayashi, K., Imanishi, K., Nishikawa, H., Ogawa, S., Katsurada, M., Nakagawa, Y. and Yoshizawa, N. (2001) Coffee consumption and serum aminotransferase in middle-aged Japanese men. J. Clin. Epidemiol., 54, 823-829.

17) Ruhl, C. E. and Everhart, J. E. (2005) Coffee and tea consumption are associated with a lower incidence of chronic liver disease in the United States. Gastroenterology, 129, 1928-1936.

18) Klatsky, A. L., Morton, C., Udaltsova, N. and Friedman, G. D. (2006) Coffee, cirrhosis, and transaminase enzymes. Arch. Intern. Med., 166, 1190-1195.

19) Bravi, F., Bosetti, C., Tavani, A., Bagnardi, V., Gallus, S., Negri, E., Franceschi, S. and La Vecchia, C. (2007) Coffee drinking and the hepatocellular carcinoma risk: a meta-analysis. Hepatology, 46, 430-435.

20) Larsson, S. C. and Wolk, A. (2007) Coffee consumption and risk of liver cancer: a meta-analysis. Gastroenterology, 132, 1740-1745.

21) Siegelman, E. S. and Roman, M. A. (2001) Imaging of hepatic steatosis. Semin. Liver Dis., 21, 71-80.

22) Kojima, S., Watanabe, N., Numata, M., Ogawa, T. and Matsuzaki, S. (2003) Increase in the prevalence of fatty liver in Japan over the past 12 years: analysis of clinical background. J. Gastroenterol., 38, 954961.

23) Nomura, H., Kashiwagi, S., Hayashi, J., Kajiyama, W., Tani, S. and Goto, M. (1988) Prevalence of fatty liver in a general population of Okinawa, Japan. Jpn. J. Med. Sci. Biol., 27, 142-149.

24) Fabbrini, E., Sullivan, S. and Klein, S. (2010) Obesity and nonalcoholic fatty liver disease: Biochemical, metabolic and clinical implications. Hepatology, 51, 679-689.

25) Weltman, M. D., Farrell, G. C., Hall, P., Ingelman-Sundberg, M. and Liddle, C. (1998) Hepatic cytochrome $\mathrm{P} 450$ 2E1 is increased in patients with nonalcoholic steatohepatitis. Hepatology, 27, 128-133.

26) Higdon, J. V. and Frei, B. (2006) Coffee and health: a review of recent human research. Crit. Rev. Food Sci. Nutr., 46, 101-123.

27) Shearer, J., Farah, A., de Paulis, T., Bracy, D. P., Pencek, R. R., Graham, T. E. and Wassermen, D. H.
(2003) Quinides of roasted coffee enhance insulin action in conscious rats. J. Nutr., 133, 3529-3532.

28) Yamauchi, R., Kobayashi, M., Matsuda, Y., Ojika, M., Shigeoka, S., Yamamoto, Y., Inoue, T., Murai, A. and Horio, F. (2010) Coffee and caffeine ameliorate hyperglycemia, fatty liver, and inflammatory adipocytokine expression in spontaneously diabetic KK-Ay mice. J. Agric. Food Chem., 58, 5597-5603.

29) Lopez-Garcia, E., van Dam, R. M., Rajpathak, S., Willett, W. C., Manson, J. E. and Hu, F. B. (2006) Changes in caffeine intake and long term weight change in men and women. Am. J. Clin. Nutr., 83, 674-680.

30) Greenberg, J. A., Boozer, C. N. and Geliebter, A. (2006) Coffee, diabetes, and weight control. Am. J. Clin. Nutr., 84, 682-693.

31) Fujioka, S., Matsuzawa, Y., Tokunaga, K. and Tarui, S. (1987) Contribution of intra-abdominal fat accumulation to the impairment of glucose and lipid metabolism in human obesity. Metabolism, 36, 5459.

32) Eguchi, Y., Eguchi, T., Mizuta, T., Ide, Y., Yasutake, T., Iwakiri, R., Hisatomi, A., Ozaki, I., Yamamoto, K., Kitajima, Y., Kawaguchi, Y., Kurose, S. and Ono, N. (2006) Visceral fat accumulation and insulin resistance are important factors in nonalcoholic fatty liver disease. J. Gastroenterol., 41, 462-469.

33) Pou, K. M., Massaro, J. M., Hoffmann, U., Vasan, R. S., Maurovich-Horvat, P., Larson, M. G., Keaney, J. F., Meigs, J. B., Lipinska, I., Kathiresan, S., Murabito, J. M., O’Donnell, C. J., Benjamin, E. J. and Fox, C. S. (2007) Visceral and subcutaneous adipose tissue volumes are cross-sectionally related to markers of inflammation and oxidative stress: the Framingham Heart Study. Circulation, 116, 12341241.

34) Williams, C. J., Fargnoli, J. L., Hwang, J. J., van Dam, R. M., Blackburn, G. L., Hu, F. B. and Mantzoros, C. S. (2008) Coffee consumption is associated with higher adiponectin concentrations in women with or without type 2 diabetes: a prospective cohort study. Diabetes Care, 31, 504-507.

35) Xu, A., Wang, Y., Keshaw, H., Xu, L. Y., Lam, K. S. and Cooper, G. L. (2003) The fat-derived hormone adiponectin alleviates alcoholic and nonalcoholic fatty liver diseases in mice. J. Clin. Invest., 112, 91-100.

36) Catalano, D., Martiline, G. F., Tonzuso, A., Pirri, C., Trovato, F. M. and Trovato, G. M. (2010) Protective role of coffee in non-alcoholic fatty liver disease (NAFLD). Dig. Dis. Sci., 55, 3200-3206.

37) Targher, G., Bertolini, L., Padovani, R., Rodella, 
S., Tessari, R., Zenari, L., Day, C. and Arcaro, G. (2007) Prevalence of nonalcoholic fatty liver disease and its association with cardiovascular disease among type 2 diabetic patients. Diabetes Care, 30, 1212-1218.

38) Tolman, K. G., Fonceca, V., Dalpiaz, A. and Tan, M. H. (2007) Spectrum of liver disease in type 2 diabetes and management of patients with diabetes and liver disease. Diabetes Care, 30, 734-743.

39) Villanova, N., Moscatiello, S., Ramilli, S., Bugianesi, D., Magalotti, D., Vanni, E., Zoli, M. and Marchesini, G. (2005) Endothelial dysfunction and cardiovascular risk profile in nonalcoholic fatty liver disease. Hepatology, 42, 473-480.

40) Nguyen-Duy, T. B., Nichaman, M. Z., Church, T. S., Blair, S. N. and Ross, R. (2003) Visceral fat and liver fat are independent predictors of metabolic risk factors in men. Am. J. Physiol. Endocrinol. Metab., 284, E1065-E1071.

41) Stefan, N., Kantartziz, K., Machann, J., Schick, F., Thamer, C., Rittig, K., Balletshofer, B., Machicao, F., Fritsche, A. and Häring, H. U. (2008) Identification and characterization of metabolically benign obesity in humans. Arch. Intern. Med., 168, 16091616. 